\title{
Spectroscopic measurements of EUV ejecta in a CME: a high-blueshift trailing thread
}

\author{
David Williams ${ }^{1}$, Deborah Baker ${ }^{1}$, Lidia van Driel-Gesztelyi ${ }^{1,2,3}$ and \\ Lucie Green ${ }^{2}$ \\ ${ }^{1}$ UCL Mullard Space Science Laboratory, UK \\ email: d.r.williams@ucl.ac.uk \\ ${ }^{2}$ Observatoire de Paris, LESIA, UMR 8109 (CNRS), France \\ ${ }^{3}$ Konkoly Observatory, Budapest, Hungary
}

\begin{abstract}
The mass of erupting prominence material can be inferred from the obscuration of emission behind this mass of cool plasma thanks to the rapid cadence of SDO/AIA images in the short EUV wavelength range (Carlyle et al. 2013, these proceedings). In comparing this approach with spectral observations from Hinode/EIS, to monitor contributions from emission seen around the erupting prominence material, we have found an intriguing component of blueshifted emission, trailing the erupting prominence, with Doppler shifts on the order of $350 \mathrm{~km} \mathrm{~s}^{-1}$ in bright lines of both He II and Fe XII.
\end{abstract}

Keywords. Sun: coronal mass ejections (CMEs), Sun: activity, Sun: prominences, Sun: UV radiation

\section{Introduction}

While observations of prominence eruptions are increasingly common with imaging instruments such as SDO/AIA, observations of these events by slit spectrometers are far more rare because of the small instantaneous field of view (IFOV), i.e., the slit size. Co-temporal, co-spatial observations from both imager and spectrometer, observing common ions are even rarer. Fortunately, Hinode/EIS and SDO/AIA each observe strong lines emitted by Fe XII and He II, and this allows us to describe not only the emission/absorption of plasma and its plane-of-sky motion, but also its line-of-sight motion where the slit IFOV overlaps with that of the imager.

\section{Observations \& Method}

A filament eruption from the south-east portion of the Sun was observed on 31-Aug2012 and observed by Hinode/EIS as it scanned its slit in 60-s exposures across a nearby portion of the off-limb corona. The compact line list contained observations of the spectrum around the Fe XII 195.12 $\AA$ and He II 256.32 $\AA$ lines. These same ions are also chiefly responsible for emission in the $193 \AA$ and $304 \AA$ bands of SDO/AIA, and so it was possible to carefully align the data of EIS to those of AIA and examine the Doppler shift of the parts of the filament that passed under the EIS slit. Conspicuously bright components of each of these lines were seen strongly blue-shifted from the mean, quasi-static off-limb components, in areas of the spectrum unoccupied by other bright lines (Brown et al. 2008; Labrosse et al. 2011), and so single-Gaussian fits to these components were made, allowing us to estimate the Doppler shift of this fast-moving material. 


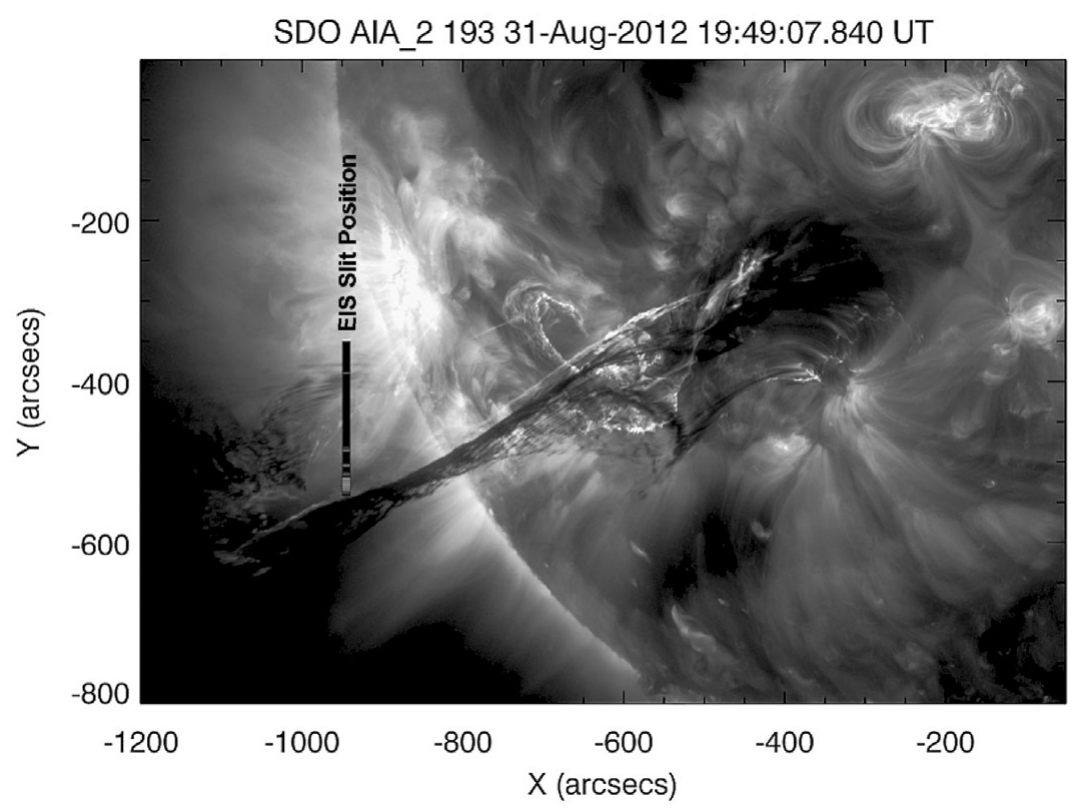

Figure 1. Hinode/EIS measurements of fitted intensity for only the blue-shifted component of Fe XII $195.12 \AA$ (overlaid on an image from SDO/AIA $193 \AA$ ). The blue-shifted component appears as a bright section of the Hinode/EIS slit (indicated), north of the dark "leg" of the prominence. This high-velocity component persists in both lines for several minutes.

\section{Results \& Summary}

The blue-shifted component of the Fe XII and He II lines (Fig. 1) marks the intersection of the slit with a thin bright feature visible in AIA, running behind the entire prominence and anchored in the flare ribbons. In both EIS lines mentioned, it is centred on positions slightly offset in $y$, indicating that temperature and velocity are a function of position. Nevertheless, the bulk motion towards the observer is supersonic $\left(v_{\text {Doppler }}>300 \mathrm{~km} \mathrm{~s}^{-1}\right)$. Since the bulk velocity of this component is non-zero, it may indicate an asymmetric outflow of material from this region behind the prominence. Co-spatial and co-temporal observations with EIS and AIA reveal a high-blueshift component of weaker emission trailing the dark prominence material of a CME. Although bright features have been noted in other EIS observations of CMEs, such high Doppler velocities have not. This rare combination of same-ion eruption data with a spectrometer and imager reveals that heating and acceleration of plasma is proceeding along and behind the erupting structure.

\section{Acknowledgement}

The research leading to these results has received funding from the EC's FP7 under grant agreement No. 284461 (eHEROES project). LvDG's is supported by Hungarian Research grant OTKA K-081421, and LG by a Royal Society University Fellowship.

\section{References}

Brown, C. M., Feldman, U., Seely, J. F., Korendyke, C. M., \& Hara, H., 2008, ApJS 176, 511

Carlyle, J., Williams, D. R., van Driel-Gesztelyi, L., \& Innes, D. E., 2013, Proceedings IAU Symposium No. 300

Labrosse, N., Schmieder, B., Heinzel, P., \& Watanabe, T., 2011, A\&3A 531, 69 